\title{
Circulating HMGB1 is elevated in veterans with Gulf War Illness and triggers the persistent pro-inflammatory microglia phenotype in male $\mathrm{C} 57 \mathrm{BI} / 6 \mathrm{~J}$ mice
}

\author{
Carla Garza-Lombó (iD) ${ }^{1}$, Morrent Thang (iD) ${ }^{1}$, Hendrik J. Greve ${ }^{1}$, Christen L. Mumaw ${ }^{1}$, Evan J. Messenger (D) ${ }^{1}$, Chandrama Ahmed ${ }^{1}$, \\ Emily Quinn ${ }^{2}$, Kimberly Sullivan ${ }^{3}$ and Michelle L. Block (iD) ${ }^{1,4 \times}$
}

(c) The Author(s) 2021

Gulf War Illness (GWI) is a chronic, multi-symptom peripheral and CNS condition with persistent microglial dysregulation, but the mechanisms driving the continuous neuroimmune pathology are poorly understood. The alarmin HMGB1 is an autocrine and paracrine pro-inflammatory signal, but the role of circulating HMGB1 in persistent neuroinflammation and GWI remains largely unknown. Using the LPS model of the persistent microglial pro-inflammatory response, male C57BI/6J mice injected with LPS $(5 \mathrm{mg} / \mathrm{kg}$ IP) exhibited persistent changes in microglia morphology and elevated pro-inflammatory markers in the hippocampus, cortex, and midbrain 7 days after LPS injection, while the peripheral immune response had resolved. Ex vivo serum analysis revealed an augmented pro-inflammatory response to LPS when microglia cells were cultured with the 7-day LPS serum, indicating the presence of bioactive circulating factors that prime the microglial pro-inflammatory response. Elevated circulating HMGB1 levels were identified in the mouse serum 7 days after LPS administration and in the serum of veterans with GWI. Tail vein injection of rHMGB1 in male C57BI/6 J mice elevated TNFa mRNA levels in the liver, hippocampus, and cortex, demonstrating HMGB1induced peripheral and CNS effects. Microglia isolated at 7 days after LPS injection revealed a unique transcriptional profile of 17 genes when compared to the acute 3 H LPS response, 6 of which were also upregulated in the midbrain by rHMGB1, highlighting a distinct signature of the persistent pro-inflammatory microglia phenotype. These findings indicate that circulating HMGB1 is elevated in GWI, regulates the microglial neuroimmune response, and drives chronic neuroinflammation that persists long after the initial instigating peripheral stimulus.

Translational Psychiatry (2021)11:390; https://doi.org/10.1038/s41398-021-01517-1

\section{INTRODUCTION}

Gulf War Illness (GWI) is a chronic multi-symptom illness with chronic central nervous system (CNS) and peripheral symptoms and pathology [1-5]. Behavioral deficits and functional brain changes have been reported in veterans with GWI, where a broad range of brain regions are affected, including the hippocampus, basal ganglia, white matter, and cortex [6-11]. Increasing evidence implicates perturbation of the peripheral immune system in both peripheral and CNS symptoms [12-26], where distinct differences in GWI-associated circulating factors are beginning to be identified $[25,27,28]$. Importantly, a recent report documented the elevation of the [(11)C]PBR28 PET signal, in areas including precuneus, prefrontal, primary motor and somatosensory cortices, demonstrating a persistent response from microglia, the resident myeloid cells in the CNS parenchyma, in veterans with GWl, long after the theater of war [29].

Microglia readily respond to events in the periphery [30], such as systemic lipopolysaccharide (LPS) administration [31], intestinal ischemia-reperfusion injury [32], liver damage [33], pulmonary injury with ozone [34], and many others. Importantly, the microglial and neuroimmune response to peripheral injury has been shown to augment ongoing neuropathology [32, 34-36], highlighting the potential importance of peripheral damage and immune dysfunction on CNS effects, particularly as a possible source of pathology in GWI.

Accumulating reports indicate that microglia have complex, function-specific phenotypes [37], where microglial dysregulation is implicated in progressive neuronal damage in diverse CNS conditions and disease [30,38]. In fact, microglia can be persistently dysregulated to drive pathology long after the instigating stimulus, such as the case with LPS [31] or chemotherapy [39], but little is understood about this persistent phenotype. The neuroimmune hypothesis of GWI $[25,27]$ holds that this persistent neuroinflammation, the chronic elevation of cytokines and reactive oxygen species in the brain that persists long after the instigating stimulus during the Gulf War, drives the associated CNS neuropathology and behavioral deficits. Several murine GWI models have exhibited microglial phenotype shifts and neuroinflammation [5, 40-42].

High-mobility group box 1 (HMGB1), a ubiquitously expressed non-histone DNA binding protein, is actively secreted by immune

\footnotetext{
${ }^{1}$ Department of Pharmacology and Toxicology, The Stark Neurosciences Research Institute, Indiana University School of Medicine, Indianapolis, IN, USA. ${ }^{2}$ Department of

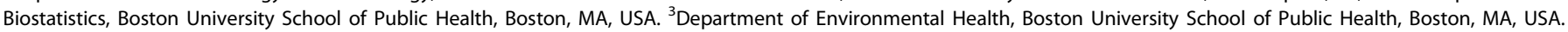
${ }^{4}$ Roudebush Veterans Affairs Medical Center, Indianapolis, IN, USA. ${ }^{\circledR}$ email: mlblock@iupui.edu
}

Received: 8 December 2020 Revised: 16 June 2021 Accepted: 28 June 2021

Published online: 12 July 2021 
cells and by damaged cells as an alarmin to function as an autocrine and paracrine signal and cytokine [43]. HMGB1 disrupts the blood-brain barrier [44] and activates cells of myeloid lineage, where HMGB1 can initiate a pro-inflammatory response in microglia, macrophages, and monocytes [45]. Peripheral HMGB1 has been linked to CNS effects, as intraperitoneal (IP) injection of HMGB1 decreases performance on memory tasks in mice [46] and induces depressive-like behavior acting as a late-phase inflammation mediator $[47,48]$. Consistent with this, several studies show HMGB1 release after systemic LPS injection [49-52], but the function of circulating HMGB1 is controversial. HMGB1 acts as a common mediator of chronic inflammatory diseases [43], where it has been found in circulation during many CNS diseases [53, 54]. Importantly, rats exposed to GWI-related chemicals pyridostigmine bromide (PB), DEET, and permethrin, in combination with moderate stress as a GWI model exhibited elevated extracellular vesicles containing $\mathrm{HMGB} 1$ in the brain and serum, supporting a potential role for circulating HMGB1 in GWI [55]. However, the specific role of circulating HMGB1 in the persistent microglial neuroinflammation response and GWI is largely unknown.

At present, there is significant mechanistic uncertainty regarding how immune dysfunction in GWI could culminate in persistent microglial responses that persist years later. To begin to address this question and start to define the basic biology of the persistent pro-inflammatory microglial (PPM) response, we employed the LPS persistent neuroinflammation model and serum from GWI veterans to explore whether: (1) Microglia fundamentally change and have a unique transcriptional signature during persistent neuroinflammation; (2) Circulating factors, like HMGB1, regulate the PPM phenotype; (3) HMGB1 is elevated in the serum of veterans with GWI when compared with healthy control veterans.

\section{MATERIALS AND METHODS \\ Animals}

C57BL/6 male mice (6-8 weeks old) were obtained from Jackson Laboratories (Bar Harbor, ME, USA). Animals were housed in an Association for Assessment and Accreditation of Laboratory Animal Care (AAALAC) official housing facility and maintained at $20-24^{\circ} \mathrm{C}$ on a $12 \mathrm{H}$ light-dark cycle Mice were individually housed in HEPA-filtered, ventilated polycarbonate cages (Lab Products, Inc., Seaford, DE) and were provided an NTP-2000 diet (Harlan Laboratories) and water ad libitum. Mice were acclimated for 1 week after delivery. All experiments were conducted with the International Care and Use Committee (IACUC) approval and the Guidelines for the Care and Use of Laboratory Animals (National Institutes of Health, Bethesda, MD, USA) were followed.

\section{LPS persistent neuroinflammation model}

Mice received a single IP injection of LPS (strain O111:B4, EMD Millipore Chemicals, Lot number $3388502,6 \mathrm{EU} / \mathrm{ng}, 5 \mathrm{mg} / \mathrm{kg}$ ) or vehicle ( $0.9 \%$ saline), which is an established murine model of persistent neuroinflammation that continues across the lifespan of the mouse [31,56], where LPS is unable to enter the brain parenchyma [31,56]. The single instigating LPS dose causes peripheral inflammation and the elevation of circulating cytokines that rapidly triggers an immediate microglial pro-inflammatory response $[57,58]$. While the initiating circulating pro-inflammatory response of the LPS administration is complete in the periphery by $24 \mathrm{H}$ after LPS administration [31], the microglial response persists for at least 1 week after the LPS treatment (LPS persistent neuroinflammation model) and can continue across the animal's lifetime to eventually culminate in behavioral deficits and neuropathology in several GWI regions, including the substantia nigra (midbrain) and hippocampus months after treatment $[31,56,58]$. While the exact pathobiology of GWI is unknown, the LPS persistent neuroinflammation model was selected because of the: brain regions affected similar to GWI [8-11], whole-body immune perturbation similar to GWI [12-25], and chronic neuroimmune consequences that persist long after the instigating stimulus, mimicking GWI [29].

Mice were humanely anesthetized with isoflurane and euthanized by exsanguination via cardiac puncture. Samples were collected at either $3 \mathrm{H}$ (acute response) or 7 days (persistent response) following LPS injection, as previously reported [57]. One cohort of mice was euthanized and microglia were isolated from the whole brain. A second cohort of mice was euthanized and the right hemisphere of each brain was fixed in a $4 \%$ paraformaldehyde solution and cryopreserved in 30\% sucrose, while the left hemisphere was dissected into GWl-affected regions (hippocampus, cortex, and midbrain) and snap frozen. The serum, liver, and spleen were also collected and either processed immediately or stored at $-80^{\circ} \mathrm{C}$. Further details are specified in the Supplementary Information.

\section{HMGB1 and TLR4 small molecule inhibitors}

Mice were treated with small molecule HMGB1 and Toll-like receptor (TLR) 4 inhibitors after the initiation of the LPS pro-inflammatory response to address their ability to ameliorate the persistent response [31] As an inhibitor of HMGB1 secretion, Inflachromene $(10 \mathrm{mg} / \mathrm{kg}$ ) [59] or vehicle (distilled water containing $5 \%$ DMSO and $40 \%$ polyethylene glycol) was administered IP daily for 6 days, beginning $24 \mathrm{H}$ after LPS injection, after the initial instigating peripheral circulating cytokine response has resolved [31]. TLR4 is a primary receptor for HMGB1 on immune cells [60]. As such, TLR4 receptor antagonist TAK-242 (3 mg/kg) [61] or vehicle (PBS containing 0.9\% DMSO) was also administered IP for 6 days, commencing $24 \mathrm{H}$ after LPS injection.

Intravenous (IV) recombinant HMGB1 protein administration Previous reports indicate that approximately $20-50 \mu \mathrm{g}$ of circulating HMGB1 can accumulate in circulation after LPS treatment in mice [62]. Mice received a single intravenous (IV) injection of carrier-free recombinant mouse HMGB1 protein (rHMGB1, $32.5 \mu \mathrm{g}$ in $200 \mu \mathrm{l})$ or vehicle $(20 \mathrm{mM}$ Tris$\mathrm{HCl}, \mathrm{pH}$ 8.0, $0.2 \mathrm{M} \mathrm{NaCl}, 1 \mathrm{mM}$ DTT) (Thermo Scientific-Invitrogen, Carlsbad, (A, USA). At $3 \mathrm{H}$ following injection, the right hemisphere of each brain was fixed in a $4 \%$ paraformaldehyde solution and cryopreserved in $30 \%$ sucrose, the left hemisphere was dissected into the hippocampus, cortex, and midbrain. The liver and spleen were also collected and either processed immediately or stored at $-80^{\circ} \mathrm{C}$.

\section{Whole-brain adult microglia isolation}

At $3 \mathrm{H}$ and 7 days post-injection, mice were anesthetized and perfused with $50 \mathrm{~mL}$ cold phosphate-buffered saline (PBS). Adult microglia were isolated from whole adult brains, as previously described [57, 63]. Further details are specified in the Supplementary Information.

\section{IBA1 immunohistochemistry}

Chromogenic microglial IBA1 histochemistry was performed as previously described $[57,63]$. Further details are specified in the Supplementary Information.

\section{Microglia morphology quantification}

To assess changes in cell volume, images of IBA 1 and fluorescent staining in the hippocampus (dentate gyrus and CA1) were acquired as Z-stacks ( $1 \mu \mathrm{m}$ steps) using a Nikon A1R Confocal microscope and a 40X objective (Nikon Instruments, Melville, NY). Seven hippocampus slices were analyzed per mouse. Hypertrophic microglia were defined as cells with a volume greater than $500 \mathrm{\mu m}^{3}$ and counted using the NIS Elements program (Nikon Instruments, Melville, NY).

\section{Enzyme-linked immunosorbent assay (ELISA)}

TNFa and HMGB1 protein were assessed in the serum with commercial ELISA kits from R\&D Systems (Minneapolis, MN, USA) and Tecan IBL International (Hamburg, Germany), according to the manufacturer's instructions.

\section{Immunology multiplex assay}

Cytokine protein levels were determined using a Milliplex assay kit (MAP Mouse Cytokine/Chemokine Magnetic Bead Panel-Immunology Multiplex Assay, \#MCYTOMAG-70K Merck-Millipore, Burlington, MA, USA) following the manufacturer's instructions.

\section{BV2: microglial cell line}

BV2 mouse microglia cell lines were acquired from NIEHS/NIH in 2002 from the John Hong lab and have been repeatedly validated in the past [64] and present. Cultures are tested for mycoplasma and the BV2 cells are negative. BV2s were cultured at $37^{\circ} \mathrm{C}$ in High-Glucose Dulbecco's Modified Eagle Medium (DMEM) supplemented with 10\% Fetal Bovine Serum (FBS), $50 \mathrm{U} /$ $\mathrm{mL}$ penicillin, and $50 \mu \mathrm{g} / \mathrm{mL}$ streptomycin in a humidified incubator with $5 \% \mathrm{CO}_{2} / 95 \%$ air. 
A.
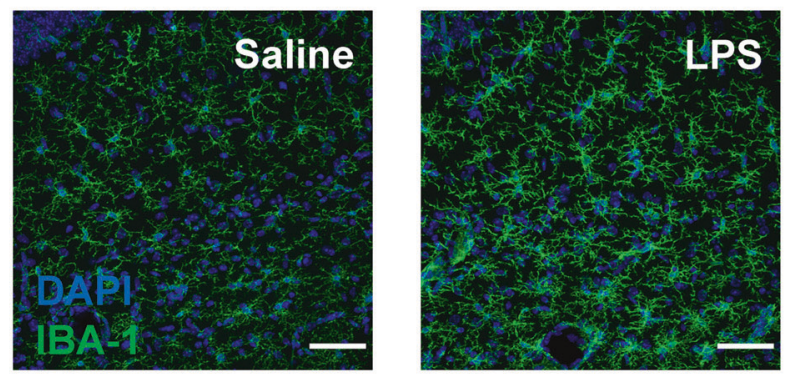

C.
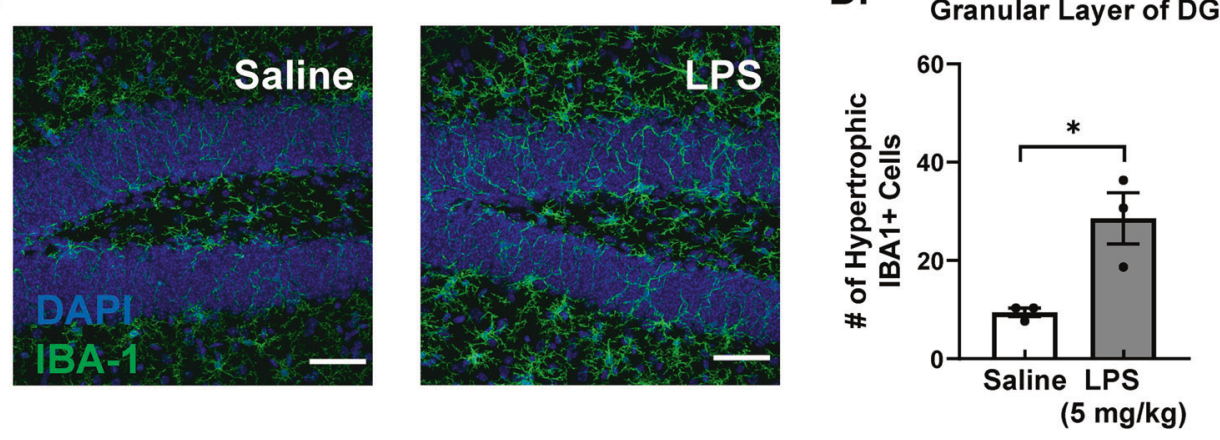

E.

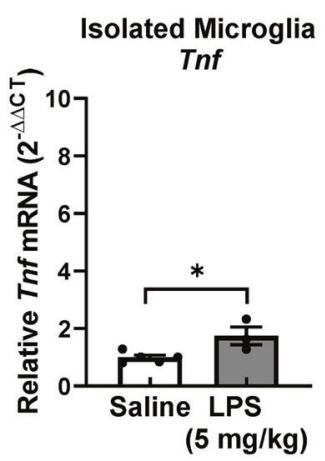

H.

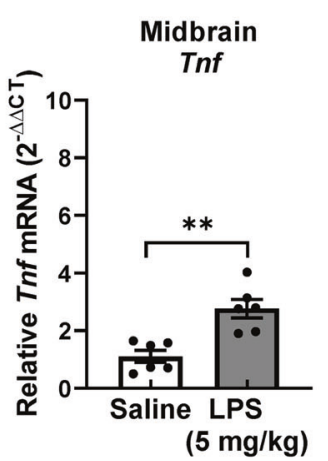

D.

B.

CA1

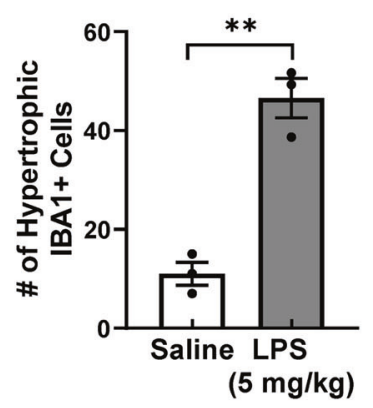

$(5 \mathrm{mg} / \mathrm{kg}$ )

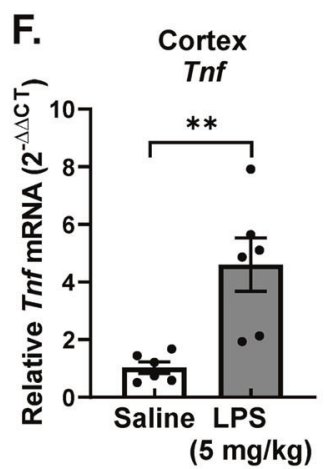

G.

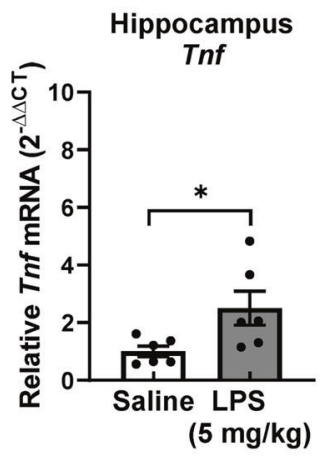

I.

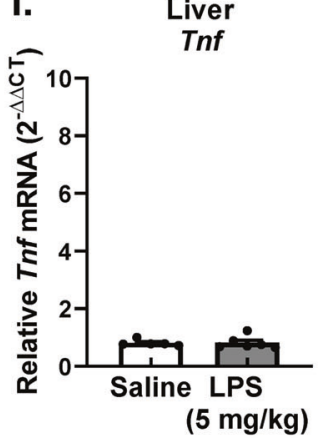

J.

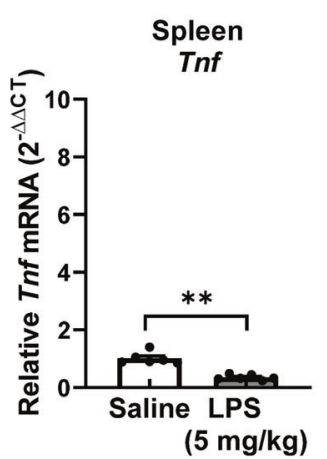

\section{Quantitative reverse transcription polymerase chain reaction} (RT-qPCR)

Total RNA was extracted from brain regions, liver, and spleen tissues with TRIzol (Thermo Scientific-Invitrogen, Carlsbad, CA, USA), following the manufacturer's instructions. RT-qPCR was performed as previously described $[57,63]$. Further details are specified in the Supplementary Information.

\section{Nanostring gene expression analysis}

RNA was extracted from the isolated microglia with a Qiagen's RNeasy Micro kit (Hilden, Germany) with on-column DNase digestion following the manufacturer's instructions. Then, $50 \mathrm{ng}$ of RNA for each sample was processed using the nCounter Mouse Neuroinflammation Panel (Seattle, WA, USA), gene expression analysis was performed on the nCounter 
Fig. 1 The persistent pro-inflammatory microglia response 7 days after LPS administration in a Murine GWI-like model. Male C57BL/6 mice received a single IP injection of LPS or saline. Microglia morphology and neuroinflammation were assessed 7 days later in this model of Gulf War Illness-like neuroimmune responses that persist long after the instigating stimulus. Immunofluorescent assessment of microglia morphology changes in the $(\mathbf{A})$ CA1 region and $(\mathbf{C})$ the granular layer of the DG was performed, where representative maximum intensity projection images taken at $\times 40$ are shown, the scale bar depicts $50 \mu \mathrm{m}$, and IBA1 (green) and DAPI (blue) are stained. The number of hypertrophic IBA $1+$ microglia cells were counted in the (B) CA1, and (D) granular layer of the DG. Hypertrophic cell $=v^{2}$ olume $>500 \mu m^{3}$. Data are reported as the mean \pm SEM. ${ }^{*} p<0.05$ and ${ }^{* *} p<0.01$ when compared to control $(n=3)$. To determine the source of the pro-inflammatory response, Tnf (TNF $\alpha)$ mRNA levels were measured in the $(\mathbf{E})$ isolated microglia, (F) cortex $(\mathbf{G})$ hippocampus, (H) midbrain, $(\mathbf{I})$ liver, and $(\mathbf{J})$ spleen by qRT-PCR and normalized to Gapdh using the $2-\Delta \Delta$ CT method. Data are reported as the mean \pm SEM. ${ }^{*} p<0.05$ and ${ }^{* *} p<0.01$ when compared to control $(n=3-6)$.

system (NanoString Technologies), and analyzed using nSolver analysis software (NanoString Technologies) according to the manufacturer's instructions. Further details are specified in the Supplementary Information.

\section{Gulf war veteran serum samples}

Serum samples from 80 Gulf War veterans (40 GWI cases, 40 healthy GW veteran controls) from the DOD funded Boston Gulf War Illness Consortium (GWIC) biorepository were used for this study. All participants provided written informed consent to participate in the study. This study was reviewed and approved by the Boston University and Indiana University institutional review board. Further details are specified in the Supplementary Information.

\begin{abstract}
Statistical analysis
The sample size was determined based on prior reports. A randomized block design was employed for the animal experiments and sample processing was performed blind, where the code denoting treatment groups was only provided for data analysis. Data are expressed as the mean \pm SEM. The ROUT method in GraphPad Prism was used to identify outliers, $Q=1$. The Levine test was used to assess the homogeneity of variance, where $p>0.05$ indicated homogeneity. Data were analyzed by analysis of variance (ANOVA) using GraphPad Prism (GraphPad Prism, San Diego, (A, USA) when the variance was homogenous. When the assumption of homogeneity of variance was not met, the Kruskal-Wallis test was used, followed by post-tests. Mean differences were analyzed by Bonferroni's post-hoc analysis or Student's $t$ test, when appropriate. A $p$ value $<0.05$ was considered statistically significant. We also conducted statistical comparisons of GWI cases vs. controls comparing serum HMGB1 levels, self-reported pyridostigmine bromide (anti-nerve agent) use, using linear regression models adjusting for age and sex [65]. The least squares means with standard error (LSM $\pm \mathrm{SE}$ ) are reported.
\end{abstract}

\section{RESULTS \\ Persistent pro-inflammatory microglia (PPM) responses without traditional circulating factors}

Circulating pro-inflammatory factors have been established as critical mediators in the initial transfer of the cytokine response in the periphery to the brain, but the identity of those that could be driving persistent neuroinflammation after this instigating stimulus is unknown. To begin to address this question, we first confirmed persistent changes in microglia morphology in the hippocampus 7 days after LPS administration, as evidenced by increased numbers of microglia with larger cell volume (hypertrophic microglia, larger than $500 \mu \mathrm{m}^{3}$ ) in the CA1 region, the granular layer of the dentate gyrus and the subgranular layer of the dentate gyrus, (Fig. 1A-D and Supplementary Fig. 1A, B, $p<0.05$ ). Increased mRNA elevation of the prototypical pro-inflammatory cytokine TNFa was also confirmed in several brain regions affected by GWI, such as the cortex, hippocampus, and midbrain, where isolated microglia from the whole brain also showed Tnf mRNA upregulation, demonstrating that microglia themselves are contributors to the persistent neuroinflammation in brain regions affected by GWI (Fig. $1 \mathrm{E}-\mathrm{H}, p<0.05$ ). These data indicate that persistent neuroinflammation occurs in brain regions that are affected in GWI 7 days after LPS-administration and that microglia themselves are a source of the persistent pro-inflammatory response, which continues long after the instigating stimulus.
Serum, liver, and the spleen are important mediators of the instigating peripheral and CNS response to LPS [66-68], but little is known about their role in the persistent immune responses. The liver exhibited no significant difference in the Tnf mRNA response 7 days after LPS administration (Fig. $11, p>0.05$ ) and the spleen showed a significantly attenuated TNFa response (Fig. $1 \mathrm{~J}, p<0.05$ ), supporting that the peripheral immune response was resolved at 7 days after the LPS administration. While most serum proinflammatory factors were unchanged 7 days after LPS administration, TNFa, RANTES, MCP-1, IL-1 $\beta$, and G-CSF were all present at significantly lower levels 7 days after LPS administration, when compared to saline-treated mice (Fig. $2 \mathrm{~A}-\mathrm{E}, p<0.05$ ). These findings indicate that at 7 days after LPS administration during the persistent microglial pro-inflammatory response, the peripheral pro-inflammatory response had resolved, including the lowering of traditional pro-inflammatory factors in circulation.

\section{Serum from the LPS persistent neuroinflammation model is bioactive}

We next sought to determine whether an unknown factor was present in the serum that could regulate microglial function and neuroinflammation from the periphery. Using a previously established ex vivo serum bioactivity assay [69], BV2 microglia cells were pretreated with $2 \%$ of serum from saline-treated mice or serum from mice treated with LPS 7 days after the injection, when neuroinflammation was present in the absence of traditional proinflammatory factors in circulation. After $30 \mathrm{~min}$, cells were then treated with either control media or $5 \mathrm{ng} / \mathrm{mL}$ LPS and supernatant was collected $3 \mathrm{H}$ later and assessed for TNFa production. Data reveal that the 7 Day LPS serum did not instigate a TNFa response on its own, but significantly augmented the microglial response to LPS (Fig. $2 \mathrm{~F}, p<0.05$ ), demonstrating that despite the absence of traditional pro-inflammatory factors, the serum primed BV2 microglia to an enhanced pro-inflammatory phenotype.

\section{Circulating HMGB1 is elevated in the LPS persistent neuroinflammation model, increased in veterans with GWI, and triggers neuroinflammation}

We chose a hypothesis directed approach to begin to address what the pro-inflammatory targets in the serum might be, as HMGB1 is known to be: elevated in the serum of diverse CNS conditions $[53,54]$, and is increased in serum exosomes of a GWI mouse model [55]. We report here that the serum HMGB1 response remains elevated at 7 days after the LPS exposure in mice (Fig. 2G, $p<0.05$ ) during the persistent neuroinflammation (Fig. 1, $p<0.05$ ). Using a similar concentration to the amount that accumulates in the murine circulation after injection of LPS [62], tail vein injection of recombinant HMGB1 $(32.5 \mu \mathrm{g})$ demonstrates that circulating $\mathrm{HMGB} 1$ triggers neuroinflammation $3 \mathrm{H}$ later, as evidenced by elevated Tnf mRNA in the hippocampus and cortex (Fig. $2 \mathrm{H}, \mathrm{I} p<0.05$ ). Interestingly, liver Tnf mRNA was significantly upregulated by rHMGB1 tail vein injection (Fig. $2 \mathrm{~J}, p<0.05$ ), but spleen Tnf mRNA was not affected (Supplementary Fig. 2). Hmgb1 mRNA expression was not altered in hippocampus, liver, and spleen (Supplementary Fig. 3A-C). Importantly, the serum from Gulf War Veterans diagnosed with GWI, as determined by the 


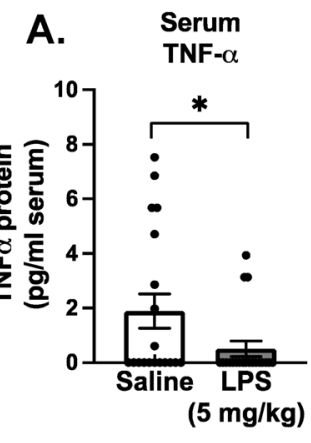

E

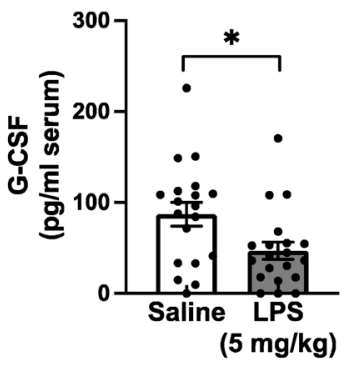

I. Cortex

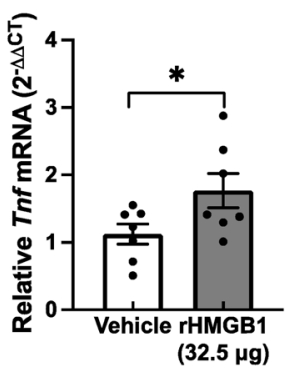

M. GW Veteran Serum

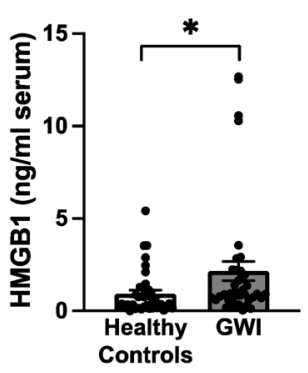

B. Serum

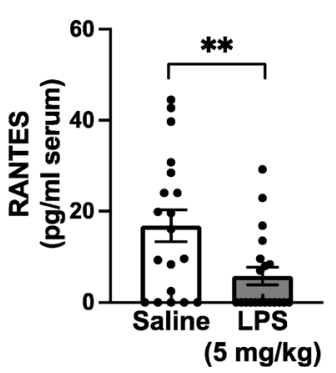

F. BV2 cells

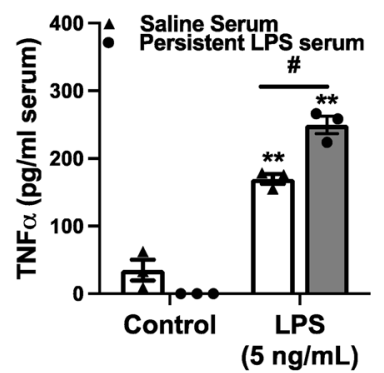

J. Liver

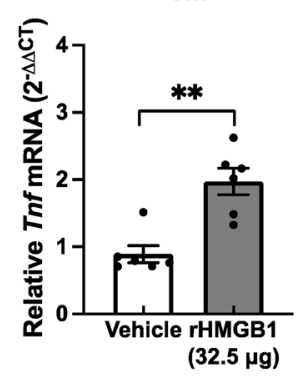

N. Midbrain

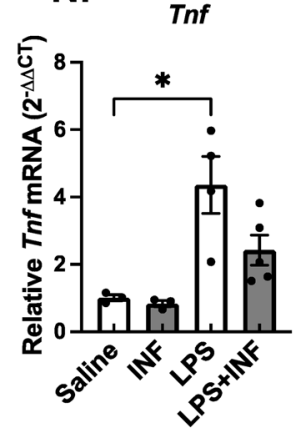

C. Serum

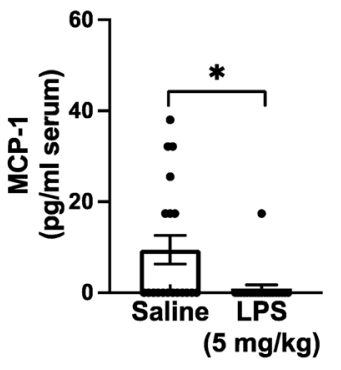

G. Serum
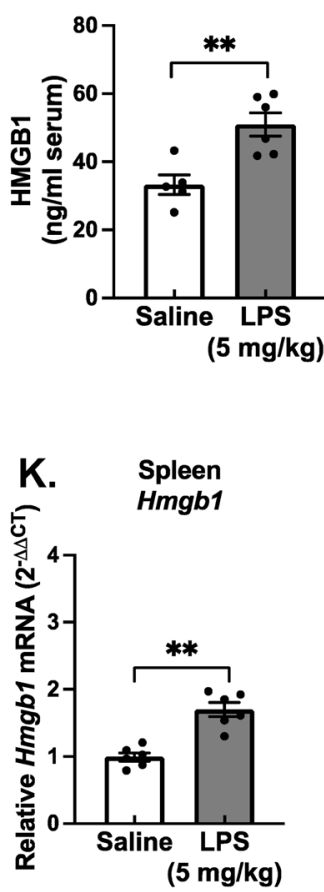

D. Serum

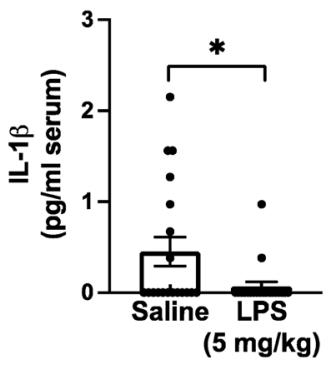

H. Hippocampus

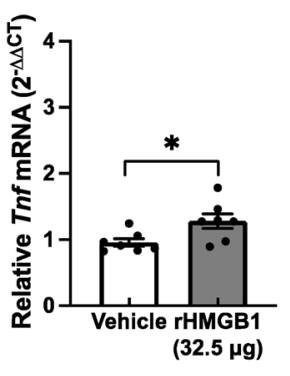

Kansas definition, exhibited significantly elevated serum HMGB1 levels as determined by ELISA, compared to healthy Gulf War Veteran controls (Fig. $2 \mathrm{M}, p<0.05$ ). There was a significant trend in the correlation between serum HMGB1 levels and self-reported exposure to pyridostigmine bromide, which was used as an prophylactic against sarin nerve gas (Supplementary Table S1, $p=0.0530$ ), further supporting that circulating HMGB1 may be generalizable to GWI-specific exposures.

Notably, Hmgb1 mRNA expression was not altered at 7 Days after LPS administration in the hippocampus, cortex, or midbrain (Supplementary Fig. 4A-C), supporting that brain parenchymal Hmgb1 gene changes were not present in the transcriptional 
Fig. 2 The LPS PPM mouse model serum has ex vivo neuroimmune bioactivity, pro-inflammatory factors below baseline, and elevated HMGB1: implications for Gulf War Illness. Male C57BL/6 mice received a single IP injection of LPS or saline and the serum was collected 7 days later in this model of Gulf War Illness- like persistent neuroinflammation. Protein levels of circulating traditional pro-inflammatory factors were determined in the serum using the Milliplex multiplex assay and ELISA: (A) TNF $\alpha$, (B) RANTES, (C) MCP-1, (D) IL1- $\beta$ and (E) G-CSF, (G) HMGB1. (F) To assess neuroimmune bioactivity ex vivo, BV2 mouse microglia cells were treated with $2 \%$ serum obtained 7 days following LPS or saline injection with media alone or LPS $(5 \mathrm{ng} / \mathrm{ml})$, where the supernatant TNF $\alpha$ was measured $3 \mathrm{H}$ later by ELISA. Data are reported as the mean \pm SEM. ${ }^{*} p<0.05$ and ${ }^{* *} p<0.01$ when compared to control and ${ }^{*} p<0.05$ when compared between exposure groups $(n=5-20)$. Hmgb1 mRNA was assessed 7 days after LPS injection in the, (K) spleen Hmgb1 mRNA, and (L) liver. To determine if circulating HMGB1 could elicit neuroinflammation, C57BL/6 mice received a single tail vein injection of rHMGB1 or vehicle and Tnf mRNA expression was assessed $3 \mathrm{H}$ later in the (H) hippocampus, (I) cortex, and (J) liver. mRNA levels were analyzed by qRT-PCR and normalized to Gapdh using the 2- $\Delta \Delta C T$ method. Data are reported as the mean \pm SEM. ${ }^{*} p<0.05$ and ${ }^{* *} p<0.01$ when compared to control $(n=3-7)$. (M) Serum samples from 40 veterans with Gulf War Illness (GWI) that had met the Kansas diagnostic for GWI and 40 healthy controls were assessed for levels of circulating HMGB1 by ELISA, Data are reported as the mean \pm SEM. ${ }^{*} p<0.05$ when compared to control $(n=40)$. To determine if HMGB1 inhibition could abolish the LPS-induced persistent neuroinflammation, at 24H after LPS injection, C57BL/6 mice were administered (N) Inflachromene (HMGB1 inhibitor, $10 \mathrm{mg} / \mathrm{kg})$ or vehicle IP or (O) TAK-242 $(3 \mathrm{mg} / \mathrm{kg}$, TLR4 inhibitor) or vehicle for 6 days and Tnf mRNA expression was assessed in the midbrain. Data are reported as the mean \pm SEM. ${ }^{*} p<0.05$ and ${ }^{* *} p<0.01$ when compared to control $(n=3-12)$.

signature of the persistent neuroinflammation. Further, there was a significant decrease in liver Hmgb1 mRNA levels (Fig. $2 \mathrm{~L}, p<0.05$ ) and an increase in the spleen (Fig. $2 \mathrm{~K}, p<0.05$ ) at 7 days after LPS administration, further highlighting the potential importance of the peripheral HMGB1 response in persistent neuroinflammation.

To further confirm that HMGB1 is regulating the persistent neuroimmune effects of LPS, we next used the small molecule inhibitors inflachromene and TAK-242, to inhibit HMGB1 secretion and activation of the HMGB1 receptor TLR4, respectively. Inhibition of HMGB1 secretion with inflachromene treatment reduced the Tnf expression comparable to control levels (Fig. $2 \mathrm{~N}$, $p<0.05)$ and TLR4 inhibition reduced the persistent neuroinflammation when compared to the LPS alone group (Fig. 2O, $p<$ 0.05). These findings support that: circulating HMGB1: can trigger neuroinflammation; is elevated during LPS-induced persistent neuroinflammation 7 days after LPS administration; regulates the LPS persistent neuroinflammation response, and is increased in Gulf War veterans diagnosed with GWI.

\section{The persistent pro-inflammatory microglia (PPM) phenotype has a unique transcriptional signature}

In an effort to define the unique transcriptional fingerprint of persistently activated microglia, we compared the distinct gene expression profiles of microglia isolated $3 \mathrm{H}$ (acute response) and 1 week (persistent response) after LPS exposure. While gene expression changed at both $3 \mathrm{H}$ (Supplementary Fig. 5A, B and Table S2) and 7 days. RNA isolated from microglia collected from mice 7 days after LPS administration demonstrated a significant, specific gene expression profile, when compared to the salinetreated group, where genes in all but the carbohydrate metabolism pathway were upregulated in the 7 days LPS group compared to the saline (Fig. 3A, $p<0.05$ ). As expected, the pathways that scored higher on this list include Inflammatory Signaling, Microglia Function, Adaptive Immune Response, Cytokine Signaling, and NF-kB (Supplementary Table S3). The volcano plot $\left(-\log _{10}\right.$ ( $p$ value) vs. fold change) in Fig. 3B shows genes differentially expressed in microglia between the saline and LPS treated mice at 7 days after treatment. The Venn diagram (Fig. 3C) illustrates that after correction, there were a total of 12 genes significantly altered by LPS treatment at $3 \mathrm{H}, 20$ genes were significantly changed by LPS at the 7 day time point, and 3 of those genes were shared between the two time points. Notably, the change in expression of only 17 genes could be attributed to only the 7 day persistent pro-inflammatory microglia phenotype. Supplementary Table S4 lists the genes significantly modified after correction at only 7 Days after LPS injection. Taken together, these data reveal that microglia during persistent neuroinflammation have a unique transcriptionally active phenotype.

Midbrain mRNA was used to confirm that the PPM cell gene expression changes occurred in the whole brain, as this GWI brain region showed an increase of Tnf gene expression and several prior reports have extensively characterized the midbrain neuroimmune pathology in LPS Persistent Neuroinflammation model $[31,56]$. RT-qPCR analysis revealed significant changes in the following persistent microglial neuroinflammation genes in the midbrain in the 7 Day LPS Persistent Neuroinflammation model: Gzma, C3, Cd72, II2rg, Cd69, Cxcl10, Ncr1, Tlr2, Sqstm1, Tnfrsf17, Cp, Cd84, Ccl5, Apoe, Anapc15, and Grn (Fig. 4A-O, $p<0.05$ ). Moreover, RT-qPCR analysis also demonstrates that midbrain mRNA from rHMGB1 treated mice exhibited upregulation of a smaller subset: Gzma, C3, Cd72, II2rg, Cd69, and Cxcl10 (Fig. 5A-F, $p<0.05$ ), demonstrating that circulating HMGB1 contributes to this transcriptional signature.

To further understand the unique PPM phenotype, the expression of the 6 PPM genes that rHMGB1 upregulates was compared in mRNA from isolated microglia from $3 \mathrm{H}$ and 7 days after LPS treatment (Fig. 5G-L, $p<0.05$ ). Notably, the $112 \mathrm{rg}, \mathrm{Cd} 69$, $\mathrm{C} x \mathrm{Cl} 10$ subset of genes were shown to peak at $3 \mathrm{H}$ and persist at lower levels of expression at the 7 Day time point after LPS administration (Fig. 5J-L, $p<0.05$ ). However, the second subset of genes Gzma, C3, and Cd72 demonstrated a significantly higher expression level at the 7 Day time point, when compared to the acute response, $3 \mathrm{H}$ after LPS administration (Fig. $5 \mathrm{G}-\mathrm{I}, p<0.05$ ). These findings demonstrate that PPM cells have a unique transcriptome, rather than just a muted chronic proinflammatory response that continues over time. While some pro-inflammatory genes did peak at the acute time point shortly after LPS administration and continue at lower levels during the persistent response, another subset of PPM genes are most robustly upregulated at the 7-day time point, supporting that the PPM cells are fundamentally changed during the process of persistent neuroinflammation.

\section{DISCUSSION}

Peripheral immune dysfunction in GWI is hypothesized to contribute to persistent microglial neuroinflammation underlying the pathobiology years after the theater of war, but the mechanisms underlying how circulating factors can culminate in persistent neuroinflammation that continues long after the instigating stimulus has been largely unknown. Here, using the LPS model of persistent neuroinflammation, we confirm that microglia specifically contribute to the persistently elevated Tnf response in the brain, when serum was devoid of traditional proinflammatory circulating cytokines. Despite the absence of these traditional circulating triggers of neuroinflammation, the serum 7 days after LPS administration was bioactive regardless, augmenting the microglial pro-inflammatory response ex vivo. The alarmin HMGB1 was discovered to be elevated in the serum of both the murine LPS model of persistent neuroinflammation and 


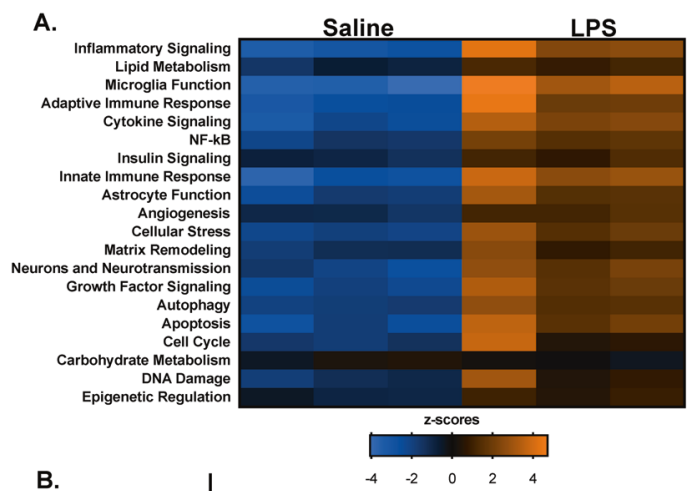

B.

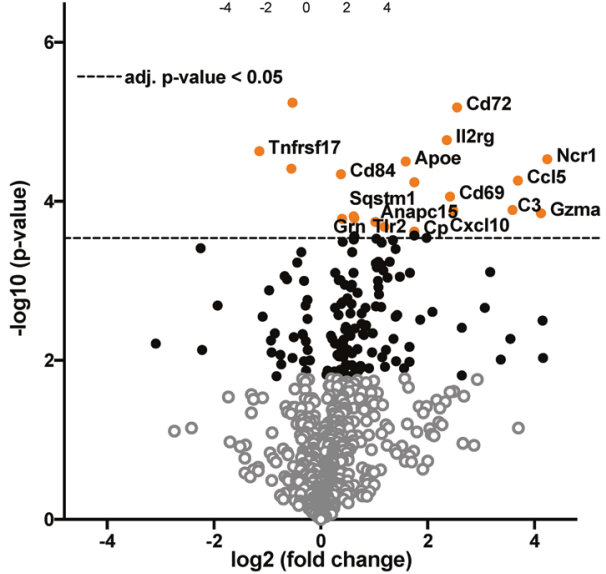

c.

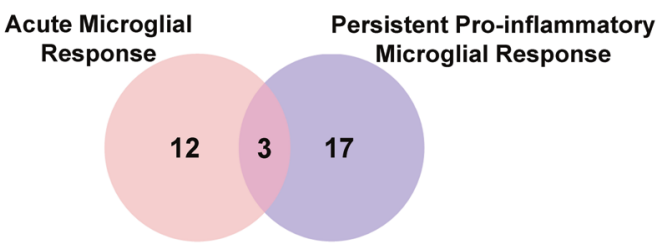

Fig. 3 The persistent pro-inflammatory microglia (PPM) transcriptome. Male C57BL/6 mice received a single IP injection of LPS or saline and microglia were isolated from whole brains at 7 days later. The nanoString Mouse Neuroinflammation Panel was used to determine differential mRNA expression. (A) Hierarchical clustering of the differentially expressed genes was assessed and significantly enriched biological pathways were identified by Ncounter system. A heatmap depicting the gene expression by category is depicted for the saline and LPS treatment at 7 days after administration. Orange indicates high expression; blue indicates low expression. (B) A volcano plots depicting the differential mRNA expression in 7 days LPS vs Saline. Values shown are the log2 (fold change) ratios of LPS treatment with respect to the saline group plotted against the $-\log 10(p$-value). The black dots denote the analyzed genes with $p$-value below the given False Discovery Rate (FDR) or $p$-value threshold. The horizontal line indicates the False Discovery Rate (FDR) threshold or adjusted $p$-value $<0.05$ determined by the Benjamini-Yekutieli and the orange dots indicate the most statistically significant genes $(p<0.05)$. (C) The Venn diagram illustrates the overlap of significantly modified genes by treatment $(n=3)$.

in veterans with GWI, but $\mathrm{Hmgb} 1$ gene expression changes were absent from the GWI-affected CNS brain regions and was instead limited to the periphery of the LPS model of persistent neuroinflammation. Mechanistically, intravenous rHMGB1 administration triggered neuroinflammation in brain regions that are affected in GWI. Microglia isolated from the LPS model of persistent neuroinflammation exhibited a unique transcriptional phenotype, where 6 of these genes were also upregulated by intravenous rHMGB1 administration. These findings provide much needed insight into how the periphery regulates the neuroimmune response outside of traditional circulating pro-inflammatory cytokines, identifies the transcriptional fingerprint of the PPM phenotype, and implicates circulating $\mathrm{HMGB} 1$ as one critical factor in the persistent microglia phenotype elevated in GWI.

Circulating cytokines are known to regulate neuroinflammation, changed circulating factors in GWI have been identified $[25,28,70]$, and circulating factors are implicated as a potential mechanism in how peripheral GWI pathology could result in CNS effects. However, a recent study documented persistent microglial activation in veterans with $\mathrm{GWI}$, as assessed by PET imaging, and showed no association of the microglial responses with the traditional pro-inflammatory circulating factors measured [71]. In the current study, using the LPS model of persistent neuroinflammation, we reveal an entire list of circulating traditional proinflammatory factors that are unnecessary for murine serum to be bioactive and regulate the microglia pro-inflammatory response (Fig. 2F). As such, these findings support that: PPM responses can be driven from the periphery, resolution of classic proinflammatory response in the periphery may not correlate with neuroinflammation or neuropathology, the traditional circulating cytokines may be misleading markers for persistent neuroinflammation, and ex vivo serum bioactivity assays with microglia may provide important insight into when peripheral pathology is regulating the neuroimmune response. Future studies should explore the feasibility of serum bioactivity assays as a potential diagnostic and therapeutic aid and also focus on the identification of other peripheral signals that can drive a PPM phenotype.

Circulating HMGB1 was identified as a mechanism of communication between the periphery and the brain to regulate the PPM response. Here, we show that the HMGB1 protein was in circulation long after the instigating single LPS IP injection (7 days, Fig. 2F), after the classic peripheral pro-inflammatory response had resolved (Fig. $2 \mathrm{~L}$ ) and during persistent neuroinflammation (Fig. 1). It is important to note that the post-translational modification of HMGB1, including redox state $[72,73]$, has a significant impact on receptor interactions driving the possible pro-inflammatory [74], chemotactic [75], or even anti-inflammatory [76] role of HMGB1, emphasizing the importance of the choice of inhibitor. Here, we note that inhibition of HMGB1 secretion and the TLR4 receptor ameliorated the persistent inflammatory effects of LPS (Fig. 2N, O), emphasizing its role in the persistent response. We also demonstrate that circulating HMGB1 is significantly elevated in veterans with GWI (Fig. $2 \mathrm{M}$ ) and associated with self-reported pyridostigmine bromide exposure (Table S5). Further, data reveal that intravenous administration of rHMGB1 into circulation in mice is sufficient to trigger the neuroinflammation response (Fig. 2H-J), including a unique microglial pro-inflammatory transcriptome (Fig. 3). Interestingly, of all tissues tested, $\mathrm{Hmgb} 1$ mRNA production was only shown to be elevated in the spleen at 7 days after LPS administration in the persistent neuroinflammation model (Fig. 2K), suggesting the periphery may be important for peripheral HMGB1 production in this model. However, at present, the peripheral source of circulating HMGB1 is unknown and should be a point of inquiry for future studies. Taken together, these findings point to circulating HMGB1 as a potential peripheral mechanism in the delayed and progressive CNS symptoms of GWI, particularly the persistent microglial response.

Accumulating evidence indicates that microglia express specific gene markers for multiple phenotypes including aging, disease, psychiatric disease, sex, viral infections, neurodegenerative diseases, and more [77]. Here, we explored whether the microglia were fundamentally changed during persistent neuroinflammation and not only do we demonstrate that these microglia exhibits a transcriptional profile 7 days after LPS administration that is unique from the acute $3 \mathrm{H}$ response (Fig. 3), but that circulating HMGB1 by itself triggers the expression of 6 of these genes in the brain (Gzma, C3, Cd72, Cxcl10, Cd69, and II2rg) (Fig. 5), where some 

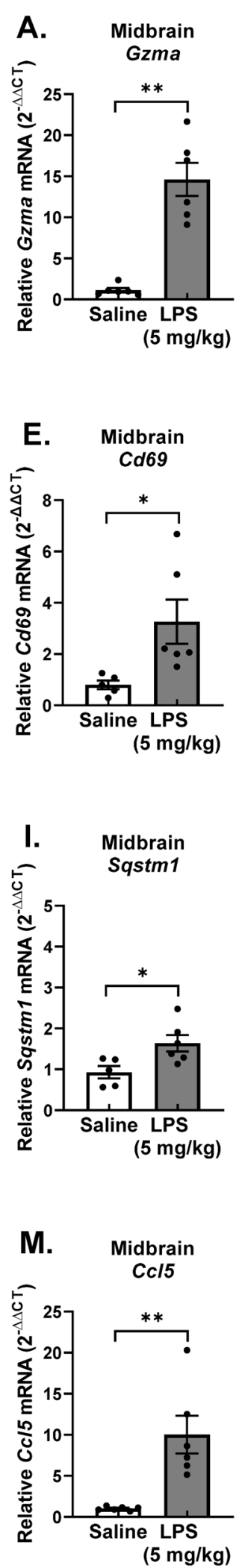
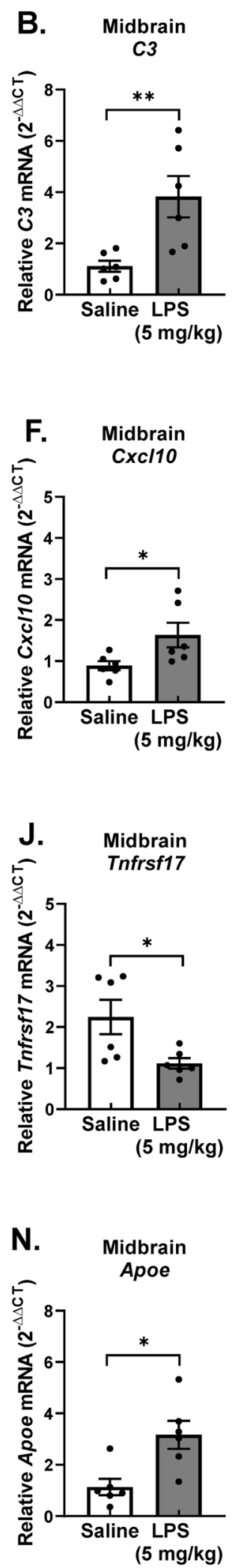
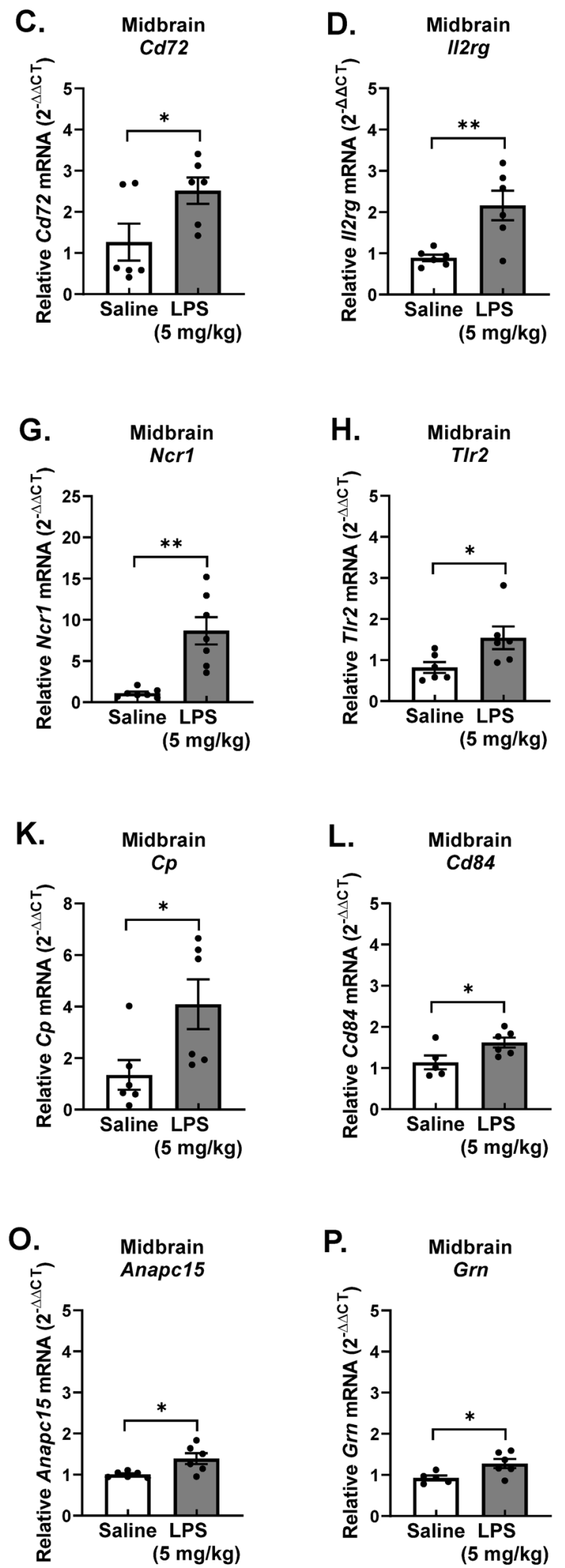

Fig. 4 Persistent pro-inflammatory microglia (PPM) gene expression in the midbrain during persistent neuroinflammation. Male $\mathrm{C} 57 \mathrm{BL} / 6$ mice received a single IP injection of LPS or saline and midbrain mRNA gene expression of PPM genes was assessed 7 days later. (A) Gzma, (B) C3, (C) Cd72, (D) II2rg, (E) Cd69, (F) Cxcl10, (G) Ncr1, (H) Tlr2, (I)Sastm1, (J) Tnfrsf17, (K) Cp, (L) Cd84, (M) Ccl15, (N) Apoe, (O) Anapc15, and (P) Grn mRNA levels were analyzed by qRT-PCR and normalized to Gapdh using the $2-\Delta \Delta C T$ method. Data are reported as the mean \pm SEM. ${ }^{*} p<0.05$ and ${ }^{* *} p<0.01$ when compared to control $(n=4-6)$. 

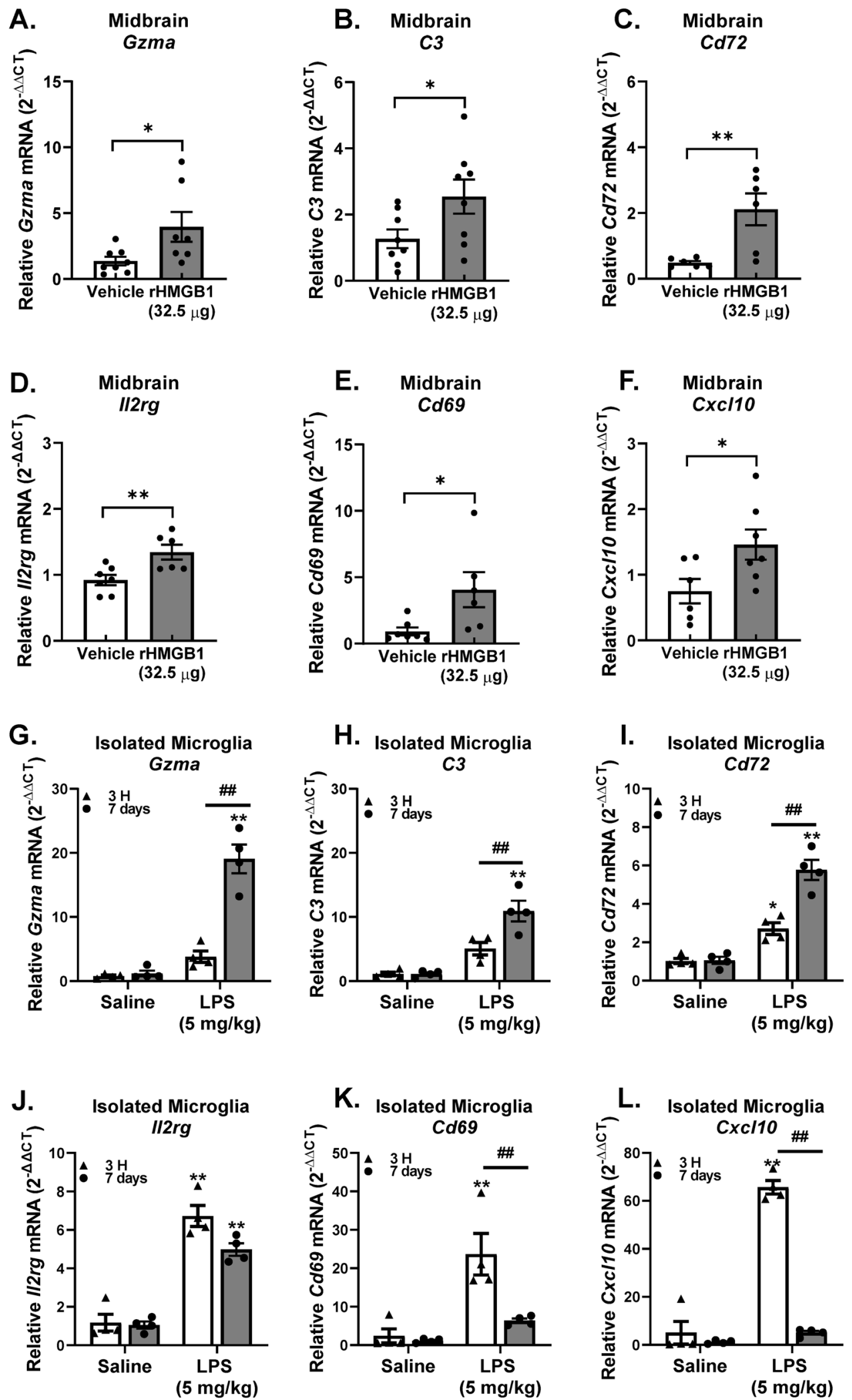

Fig. 5 Intravenous HMGB1 administration elevates persistent pro-inflammatory microglia (PPM) genes in the midbrain. C57BL/6 mice received a single IV injection of recombinant HMGB1 protein ( $\mathrm{rHMGB1}, 32.5 \mu \mathrm{g}$ ) or vehicle and mRNA was assessed in the midbrain $3 \mathrm{H}$ later. PPM transcriptome genes (A) Gzma, (B) C3, (C) Cd72, (D) $1 / 2 \mathrm{rg}$, (E) Cd69, and (F) Cxcl10 mRNA levels were measured in the midbrain. Male C57BL/ 6 mice received a single IP injection of LPS or saline and neuroinflammation was assessed $3 \mathrm{H}$ or 7 days later and PPM gene expression levels were compared: (G) Gzma, (H) C3, (I) Cd72, (J) II2rg, (K) Cd69, and (L) Cxcl10 mRNA levels were measured in the isolated microglia. mRNA levels were analyzed by qRT-PCR and normalized to Gapdh using the $2-\Delta \Delta C T$ method. Data are reported as the mean \pm SEM. ${ }^{*} p<0.05$ and ${ }^{* *} p<0.01$ when compared to control $(n=4-6)$. 


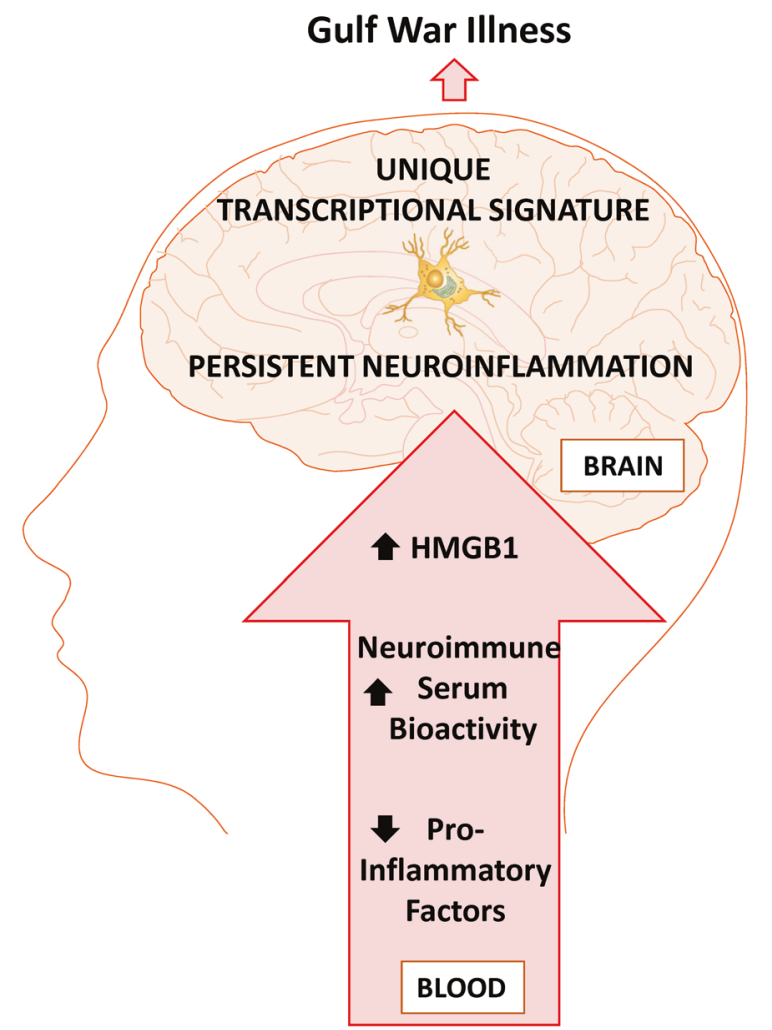

GWI-LIKE PERIPHERAL IMMUNE DYSREGULATION

Fig. 6 Circulating HMGB1 is elevated in Gulf War Illness and drives persistent neuroinflammation. Peripheral immune perturbation from IP LPS administration in the murine LPS Persistent Neuroinflammation model can mimic peripheral immune dysregulation similar to GWI that results in persistent neuroimmune bioactive serum that can modify the microglial pro-inflammatory response, long after the prototypical pro-inflammatory response in the serum has resolved. Inhibition of HMGB1 attenuates the persistent pro-inflammatory response in the brain and administration of recombinant HMGB1 can trigger neuroinflammation. Circulating HMGB1 is upregulated in the serum of Gulf War Veterans and in the bioactive serum of mice during the LPS-induced persistent neuroinflammation. Isolated microglia reveal a unique Persistent Pro-inflammatory Microglia transcriptome that is triggered in part by HMGB1, which may provide important insight into the persistent nature of neuroinflammation in GWI.

of these factors have been associated with GWI and GWI-like symptoms. For example, the C3 gene encodes for complement C3 and is not only a critical component of the complement system, but it is: activated in human AD brains, required for neurodegeneration in mouse models of amyloidosis and tauopathy [78], and has been previously shown to be upregulated in the cortex of a mice model of GWI [55]. CXCL10 (IP-10) is a small-molecularweight pro-inflammatory chemokine produced by many cells, including microglia [79] and the CXCL10 protein was found upregulated in GWI serum and serum from myalgic encephalomyelitis patients [21]. While we found an increase in Cxcl10 gene expression 7 days after LPS administration in the mouse model (Fig. 4F), we did not see any significant changes in the serum levels of the CXCL10 (IP-10) protein. We also show that Cd72 is elevated in response to HMGB1-induced persistent neuroinflammation (Fig. 4C), where $C d 72$ is a receptor expressed on microglia [80] and is a gene commonly upregulated in the brain after repeated LPS administration [81]. However, at present, there are no other reports testing the association of $C d 72$ with GWI models nor GWI. In addition, CD69 is type II C-lectin membrane bound receptor and a marker of lymphocyte activation [82] that is expressed in a subset of microglia in the CK-p25 inducible mouse model of severe neurodegeneration [83]. Although we found an increase in Cd69 gene expression 7 days after LPS administration in the mouse model (Fig. 4E), its role in GWI has not yet been explored outside of the current study and its function in microglia is poorly understood. Interleukin 2 receptor gamma (II2rg) is an important signaling component for many interleukin receptors expressed on microglia [71], where despite the upregulation in gene expression we show in response to persistent neuroinflammation (Fig. 4D) and HMGB1 (Fig. 5D), the implications for this CNS or peripheral immune gene expression in GWI are also unknown. Finally, while Gzma, the gene for Granzyme A, is expressed in myeloid cells [84], it's expression in microglia specifically and role in GWI has yet to be explored. Importantly, examining the pattern of expression of these 6 PPM genes over time revealed that some PPM genes peaked early at the acute $3 \mathrm{H}$ time point shortly after LPS administration and continued on at lower levels during the persistent response. However, another subset of PPM genes had the largest increase at the 7 day time point, demonstrating a unique transcriptional fingerprint for PPM cells. These findings may provide important insight into the mechanisms underlying persistent neuroinflammation that continues long after the initial stimulus and may provide much needed insight into potential GWI biomarkers and therapeutic targets.

In summary, GWI presents as a whole body illness and how the periphery could regulate the persistent CNS neuroimmune response in GWI is poorly understood. In the current study, data show that after peripheral immune perturbation with LPS, circulating factors present in the serum, independent of traditional pro-inflammatory factors, have neuroimmune bioactivity and regulate the microglial response ex vivo. Data also reveal that HMGB1 is present in this LPS persistent neuroinflammation model 7 days after LPS administration, during the persistent neuroinflammation and after the peripheral immune response has resolved. Importantly, HMGB1 was also elevated in serum from Gulf War veterans diagnosed with GWl, compared to healthy controls, further supporting the importance of circulating HMGB1 for GWI. Tail vein injection of rHMGB1 in mice confirmed that circulating HMGB1 triggers neuroinflammation. Further analysis of microglia isolated from the LPS persistent neuroinflammation GWl-like model characterized the fundamental neuroimmune changes that occurred in these cells and identified a unique microglia transcriptome, where rHMGB1 tail vein injection also specifically elevated 6 of these genes (Gzma, C3, Cd72, Cxcl10, $C d 69$, and $112 \mathrm{rg})$ in the midbrain. Taken together, these findings point to circulating HMGB1 as a critical mechanism underlying how the periphery in GWI might regulate the persistent microglial response and identify novel potential biomarkers, unique therapeutic targets, and a serum bioactivity assay to elucidate the pathobiology and target future treatment in GWI (Fig. 6).

\section{DATA AVAILABILITY}

The datasets generated during and/or analyzed during the current study are available from the corresponding author on reasonable request and the Nanostring data is uploaded as a supplemental file.

\section{REFERENCES}

1. Cooper CM, Briggs RW, Farris EA, Bartlett J, Haley RW, Odegard TN. Memory and functional brain differences in a national sample of U.S. veterans with Gulf War Illness. Psychiatry Res. 2016;250:33-41.

2. White RF, Steele L, O'Callaghan JP, Sullivan K, Binns JH, Golomb BA, et al. Recent research on Gulf War Illness and other health problems in veterans of the 1991 Gulf War: Effects of toxicant exposures during deployment. Cortex. 2016;74:449-75. 
3. Committee on Gulf War and Health. In: Cory-Slechta D, Wedge R, editors. Gulf war and health: volume 10: update of health effects of serving in the gulf war 2016. Washington, DC: National Academies Press; 2016.

4. Abdullah L, Crynen G, Reed J, Bishop A, Phillips J, Ferguson S, et al. Proteomic CNS profile of delayed cognitive impairment in mice exposed to Gulf War agents. Neuromolecular Med. 2011;13:275-88.

5. Ojo JO, Abdullah L, Evans J, Reed JM, Montague H, Mullan MJ, et al. Exposure to an organophosphate pesticide, individually or in combination with other Gulf War agents, impairs synaptic integrity and neuronal differentiation, and is accompanied by subtle microvascular injury in a mouse model of Gulf War agent exposure. Neuropathology. 2014;34:109-27.

6. Tillman GD, Calley CS, Buhl VI, Chiang HS, Haley RW, Hart J Jr, et al. Electrophysiological correlates of semantic memory retrieval in Gulf War Syndrome 2 patients. J Neurol Sci. 2017;373:66-72.

7. Turner MP, Hubbard NA, Himes LM, Faghihahmadabadi S, Hutchison JL, Bennett IJ, et al. Cognitive slowing in Gulf War Illness predicts executive network hyperconnectivity: study in a population-representative sample. NeuroimageClin. 2016;12:535-41.

8. Engdahl BE, James LM, Miller RD, Leuthold AC, Lewis SM, Carpenter AF, et al. A Magnetoencephalographic (MEG) Study of Gulf War Illness (GWI). Ebiomedicine. 2016;12:127-32.

9. Cooper CM, Briggs RW, Farris EA, Bartlett J, Haley RW, Odegard TN. Memory and functional brain differences in a national sample of US veterans with Gulf War Illness. Psychiat Res-Neuroim. 2016;250:33-41.

10. Menon PM, Nasrallah HA, Reeves RR, Ali JA. Hippocampal dysfunction in Gulf War Syndrome. A proton MR spectroscopy study. Brain Res. 2004;1009:189-94.

11. Odegard TN, Cooper CM, Farris EA, Arduengo J, Bartlett J, Haley R. Memory impairment exhibited by veterans with Gulf War Illness. Neurocase. 2013;19:316-27.

12. Smylie AL, Broderick G, Fernandes $H$, Razdan S, Barnes Z, Collado F, et al. A comparison of sex-specific immune signatures in Gulf War Illness and chronic fatigue syndrome. BMC Immunol. 2013;14:29.

13. Broderick G, Kreitz A, Fuite J, Fletcher MA, Vernon SD, Klimas N. A pilot study of immune network remodeling under challenge in Gulf War Illness. Brain Behav Immun. 2011;25:302-13.

14. Vojdani A, Thrasher JD. Cellular and humoral immune abnormalities in Gulf War veterans. Environ Health Perspect. 2004;112:840-846.

15. Skowera A, Hotopf M, Sawicka E, Varela-Calvino R, Unwin C, Nikolaou V, et al. Cellular immune activation in Gulf War veterans. J Clin Immunol. 2004;24:66-73.

16. Whistler $T$, Fletcher $M A$, Lonergan $W$, Zeng XR, Lin JM, Laperriere $A$, et al. Impaired immune function in Gulf War Illness. BMC Med Genomics. 2009;2:12.

17. Johnson GJ, Slater BC, Leis LA, Rector TS, Bach RR. Blood Biomarkers of Chronic Inflammation in Gulf War Illness. PLoS ONE. 2016;11:e0157855.

18. Zhang Q, Zhou XD, Denny T, Ottenweller JE, Lange G, LaManca JJ, et al. Changes in immune parameters seen in Gulf War veterans but not in civilians with chronic fatigue syndrome. Clin Diagn Lab Immunol. 1999;6:6-13.

19. Broderick G, Fletcher MA, Gallagher M, Barnes Z, Vernon SD, Klimas NG. Exploring the diagnostic potential of immune biomarker coexpression in Gulf War Illness. Methods Mol Biol. 2012;934:145-64.

20. Maloney SR, Jensen S, Gil-Rivas V, Goolkasian P. Latent viral immune inflammatory response model for chronic multisymptom illness. Med Hypotheses. 2013;80:220-229.

21. Khaiboullina SF, DeMeirleir KL, Rawat S, Berk GS, Gaynor-Berk RS, Mijatovic T, et al. Cytokine expression provides clues to the pathophysiology of Gulf War Illness and myalgic encephalomyelitis. Cytokine. 2015;72:1-8.

22. Parkitny L, Middleton S, Baker K, Younger J. Evidence for abnormal cytokine expression in Gulf War Illness: A preliminary analysis of daily immune monitoring data. BMC Immunol. 2015;16:57.

23. Broderick G, Ben-Hamo R, Vashishtha S, Efroni S, Nathanson L, Barnes Z, et al. Altered immune pathway activity under exercise challenge in Gulf War Illness: an exploratory analysis. Brain Behav Immun. 2013;28:159-69.

24. Georgopoulos AP, James LM, Mahan MY, Joseph J, Georgopoulos A, Engdahl BE. Reduced Human Leukocyte Antigen (HLA) Protection in Gulf War Illness (GWI). Ebiomedicine. 2016;3:79-85.

25. Abou-Donia MB, Conboy LA, Kokkotou E, Jacobson E, Elmasry EM, Elkafrawy $P$, et al. Screening for novel central nervous system biomarkers in veterans with Gulf War Illness. Neurotoxicol Teratol. 2017;61:36-46.

26. Abou-Donia MB, et al. Using plasma autoantibodies of central nervous system proteins to distinguish veterans with Gulf War Illness from healthy and symptomatic controls. Brain Sci. 2020;10:610.

27. O'Callaghan JP, Michalovicz LT, Kelly KA. Supporting a neuroimmune basis of Gulf War Illness. Ebiomedicine. 2016;13:5-6.

28. Emmerich T, Zakirova Z, Klimas N, Sullivan K, Shetty AK, Evans JE, et al. Phospholipid profiling of plasma from GW veterans and rodent models to identify potential biomarkers of Gulf War Illness. PLoS ONE. 2017;12:e0176634.
29. Alshelh Z, Albrecht DS, Bergan C, Akeju O, Clauw DJ, Conboy L, et al. In-vivo imaging of neuroinflammation in veterans with Gulf War illness. Brain Behav Immun. 2020;87:498-507.

30. Hickman S, Izzy S, Sen P, Morsett L, El Khoury J. Microglia in neurodegeneration Nat Neurosci. 2018;21:1359-69.

31. Qin L, Wu X, Block ML, Liu Y, Breese GR, Hong JS, et al. Systemic LPS causes chronic neuroinflammation and progressive neurodegeneration. Glia. 2007;55:453-62.

32. Zhou J, Huang WQ, Li C, Wu GY, Li YS, Wen SH, et al. Intestinal ischemia/reperfusion enhances microglial activation and induces cerebral injury and memory dysfunction in rats. Crit Care Med. 2012;40:2438-48.

33. Butterworth RF. Neuroinflammation in acute liver failure: mechanisms and novel therapeutic targets. Neurochem Int. 2011;59:830-836.

34. Mumaw $\mathrm{CL}$, et al. Microglial priming through the lung-brain axis: the role of air pollution-induced circulating factors. FASEB J. 2016;30:1880-91.

35. Lacagnina MJ, et al. A role for neuroimmune signaling in a rat model of Gulf War Illness-related pain. Brain Behav Immun. 2020;91:418-28.

36. Bose D, et al. Obesity worsens Gulf War Illness symptom persistence pathology by linking altered gut microbiome species to long-term gastrointestinal, hepatic, and neuronal inflammation in a mouse model. Nutrients. 2020;12:2764.

37. Hammond TR, Dufort C, Dissing-Olesen L, Giera S, Young A, Wysoker A, et al. Single-cell RNA sequencing of microglia throughout the mouse lifespan and in the injured brain reveals complex cell-state changes. Immunity. 2019;50:253-71 e256.

38. Block ML, Zecca L, Hong JS. Microglia-mediated neurotoxicity: uncovering the molecular mechanisms. Nat Rev Neurosci. 2007;8:57-69.

39. Gibson EM, Nagaraja S, Ocampo A, Tam LT, Wood LS, Pallegar PN, et al. Methotrexate chemotherapy induces persistent tri-glial dysregulation that underlies chemotherapy-related cognitive impairment. Cell. 2019;176:43-55 e13.

40. O'callaghan JP, Kelly KA, Locker AR, Miller DB, Lasley SM. Corticosterone primes the neuroinflammatory response to DFP in mice: potential animal model of Gulf War Illness. J Neurochem. 2015;133:708-21.

41. Parihar VK, Hattiangady B, Shuai B, Shetty AK. Mood and memory deficits in a model of Gulf War Illness are linked with reduced neurogenesis, partial neuron loss, and mild inflammation in the hippocampus. Neuropsychopharmacology. 2013;38:2348-62.

42. Abdullah L, Evans JE, Joshi U, Crynen G, Reed J, Mouzon B, et al. Translational potential of long-term decreases in mitochondrial lipids in a mouse model of Gulf War Illness. Toxicology. 2016;372:22-33.

43. Harris HE, Andersson U, Pisetsky DS. HMGB1: a multifunctional alarmin driving autoimmune and inflammatory disease. Nat Rev Rheumatol. 2012;8:195-202.

44. Zhang J, Takahashi HK, Liu K, Wake H, Liu R, Maruo T, et al. Anti-high mobility group box-1 monoclonal antibody protects the blood-brain barrier from ischemia-induced disruption in rats. Stroke. 2011;42:1420-1428.

45. Gao HM, Zhou H, Zhang F, Wilson BC, Kam W, Hong JS. HMGB1 acts on microglia Mac1 to mediate chronic neuroinflammation that drives progressive neurodegeneration. J Neurosci. 2011;31:1081-92.

46. Vacas S, Degos V, Tracey KJ, Maze M. High-mobility group box 1 protein initiates postoperative cognitive decline by engaging bone marrow-derived macrophages. Anesthesiology. 2014;120:1160-1167.

47. Lian YJ, Gong H, Wu TY, Su WJ, Zhang Y, Yang YY, et al. Ds-HMGB1 and fr-HMGB induce depressive behavior through neuroinflammation in contrast to nonoxidHMGB1. Brain Behav Immun. 2017;59:322-32.

48. Wu TY, Liu L, Zhang W, Zhang Y, Liu YZ, Shen XL, et al. High-mobility group box-1 was released actively and involved in LPS induced depressive-like behavior. J Psychiatr Res. 2015;64:99-106.

49. O'connor KA, Hansen MK, Rachal Pugh C, Deak MM, Biedenkapp JC, Milligan ED, et al. Further characterization of high mobility group box 1 (HMGB1) as a proinflammatory cytokine: central nervous system effects. Cytokine. 2003;24:254-65.

50. Li W, Deng M, Loughran PA, Yang M, Lin M, Yang C, et al. LPS induces active HMGB1 release from hepatocytes into exosomes through the coordinated activities of TLR4 and Caspase-11/GSDMD signaling. Front Immunol. 2020;11:229.

51. Abdulmahdi W, Patel $D$, Rabadi MM, Azar T, Jules E, Lipphardt $M$, et al. HMGB1 redox during sepsis. Redox Biol. 2017;13:600-607.

52. Hasegawa A, Iwasaka H, Hagiwara S, Noguchi T. Relationship between HMGB1 and tissue protective effects of HSP72 in a LPS-induced systemic inflammation model. J Surg Res. 2011;169:85-91.

53. Festoff BW, Sajja RK, van Dreden P, Cucullo L. HMGB1 and thrombin mediate the blood-brain barrier dysfunction acting as biomarkers of neuroinflammation and progression to neurodegeneration in Alzheimer's disease. J Neuroinflammation. 2016;13:194.

54. Baran A, Bulut M, Kaya MC, Demirpençe Ö, Sevim B, Akıl E, et al. High-sensitivity C-reactive protein and high mobility group box-1 levels in Parkinson's disease. Neurol Sci. 2019;40:167-73. 
55. Madhu LN, Attaluri S, Kodali M, Shuai B, Upadhya R, Gitai D, et al. Neuroinflammation in Gulf War Illness is linked with HMGB1 and complement activation, which can be discerned from brain-derived extracellular vesicles in the blood. Brain Behav Immun. 2019;81:430-43.

56. Qin L, Liu Y, Hong JS, Crews FT. NADPH oxidase and aging drive microglial activation, oxidative stress, and dopaminergic neurodegeneration following systemic LPS administration. Glia. 2013;61:855-68.

57. Taetzsch T, Levesque S, McGraw C, Brookins S, Luqa R, Bonini MG, et al. Redox regulation of NF-kappaB p50 and $\mathrm{M} 1$ polarization in microglia. Glia. 2015;63:423-40.

58. Catorce MN, Gevorkian G. LPS-induced murine neuroinflammation model: main features and suitability for pre-clinical assessment of nutraceuticals. Curr Neuropharmacol. 2016;14:155-64.

59. Lee S, Nam Y, Koo JY, Lim D, Park J, Ock J, et al. A small molecule binding HMGB1 and HMGB2 inhibits microglia-mediated neuroinflammation. Nat Chem Biol. 2014;10:1055-60.

60. Yu M, Wang H, Ding A, Golenbock DT, Latz E, Czura CJ, et al. HMGB1 signals through toll-like receptor (TLR) 4 and TLR2. Shock. 2006;26:174-179.

61. Ono Y, Maejima Y, Saito M, Sakamoto K, Horita S, Shimomura K, et al. TAK-242, a specific inhibitor of Toll-like receptor 4 signalling, prevents endotoxemia-induced skeletal muscle wasting in mice. Sci Rep. 2020;10:694.

62. Wang $\mathrm{H}$, Bloom $\mathrm{O}$, Zhang $\mathrm{M}$, Vishnubhakat JM, Ombrellino M, Che J, et al. HMG-1 as a late mediator of endotoxin lethality in mice. Science. 1999;285:248-51.

63. Harms AS, Tansey MG. Isolation of murine postnatal brain microglia for phenotypic characterization using magnetic cell separation technology. Methods Mol Biol. 2013;1041:33-39.

64. Pei Z, Pang H, Qian L, Yang S, Wang T, Zhang W, et al. MAC1 mediates LPSinduced production of superoxide by microglia: the role of pattern recognition receptors in dopaminergic neurotoxicity. Glia. 2007;55:1362-73.

65. Noble WS. How does multiple testing correction work? Nat Biotechnol. 2009;27:1135-1137.

66. Suzuki S, Nakamura S, Serizawa A, Sakaguchi T, Konno H, Muro H, et al. Role of Kupffer cells and the spleen in modulation of endotoxin-induced liver injury after partial hepatectomy. Hepatology. 1996;24:219-25.

67. Rosas-Ballina M, Ochani M, Parrish WR, Ochani K, Harris YT, Huston JM, et al. Splenic nerve is required for cholinergic antiinflammatory pathway control of TNF in endotoxemia. Proc Natl Acad Sci USA. 2008;105:11008-13.

68. Huston JM, Ochani M, Rosas-Ballina M, Liao H, Ochani K, Pavlov VA, et al. Splenectomy inactivates the cholinergic antiinflammatory pathway during lethal endotoxemia and polymicrobial sepsis. J Exp Med. 2006;203:1623-1628.

69. Mumaw CL, Levesque S, McGraw C, Robertson S, Lucas S, Stafflinger JE, et al. Microglial priming through the lung-brain axis: the role of air pollution-induced circulating factors. Faseb J. 2016;30:1880-91.

70. Joshi U, Pearson A, Evans JE, Langlois $H$, Saltiel N, Ojo J, et al. A permethrin metabolite is associated with adaptive immune responses in Gulf War Illness. Brain Behav Immun. 2019:81:545-59.

71. Zhao X, Liao Y, Morgan S, Mathur R, Feustel P, Mazurkiewicz J, et al. Noninflammatory changes of microglia are sufficient to cause epilepsy. Cell Rep. 2018;22:2080-93.

72. Kwak MS, Kim HS, Lee B, Kim YH, Son M, Shin JS. Immunological significance of HMGB1 post-translational modification and redox biology. Front Immunol. 2020;11:1189.

73. Aucott H, Lundberg J, Salo H, Klevenvall L, Damberg P, Ottosson L, et al. Neuroinflammation in Response to Intracerebral Injections of Different HMGB1 Redox Isoforms. J Innate Immun. 2018;10:215-27.

74. Manivannan S, Marei O, Elalfy O, Zaben M. Neurogenesis after traumatic brain injury-the complex role of HMGB1 and neuroinflammation. Neuropharmacology. 2021;183:108400.

75. De Leo F, Quilici G, Tirone M, De Marchis F, Mannella V, Zucchelli C, et al. Diflunisal targets the HMGB1/CXCL12 heterocomplex and blocks immune cell recruitment. EMBO Rep. 2019;20:e47788.

76. Li L, Lu YQ. The Regulatory Role of High-Mobility Group protein 1 in sepsis-related immunity. Front Immunol. 2020;11:601815.

77. Gerrits E, Heng Y, Boddeke E, Eggen BJL. Transcriptional profiling of microglia; current state of the art and future perspectives. Glia. 2020;68:740-55.
78. Wu T, Dejanovic B, Gandham VD, Gogineni A, Edmonds R, Schauer S, et al. Complement $\mathrm{C} 3$ Is activated in human $A D$ brain and is required for neurodegeneration in mouse models of amyloidosis and tauopathy. Cell Rep. 2019;28:2111-23 e2116.

79. Plastira I, Bernhart E, Joshi L, Koyani CN, Strohmaier H, Reicher H, et al. MAPK signaling determines lysophosphatidic acid (LPA)-induced inflammation in microglia. J Neuroinflammation. 2020;17:127.

80. Tsuchihashi R, Sawano T, Watanabe F, Yamaguchi N, Yamaguchi W, Niimi K, et al. Upregulation of IFN-beta induced by Sema4D-dependent partial Erk1/2 inhibition promotes NO production in microglia. Biochem Biophys Res Commun. 2020;521:827-32.

81. Bodea LG, Wang Y, Linnartz-Gerlach B, Kopatz J, Sinkkonen L, Musgrove R, et al. Neurodegeneration by activation of the microglial complement-phagosome pathway. J Neurosci. 2014;34:8546-56.

82. Cibrian D, Sanchez-Madrid F. CD69: from activation marker to metabolic gatekeeper. Eur J Immunol. 2017;47:946-53.

83. Mathys H, Adaikkan C, Gao F, Young JZ, Manet E, Hemberg M, et al. Temporal tracking of microglia activation in neurodegeneration at single-cell resolution. Cell Rep. 2017;21:366-80.

84. Hartmann W, Marsland BJ, Otto B, Urny J, Fleischer B, Korten S. A novel and divergent role of granzyme $A$ and $B$ in resistance to helminth infection. J Immunol. 2011;186:2472-81.

\section{ACKNOWLEDGEMENTS}

This study was funded by NIH RO1 ES029835GW and graciously supported by $\mathrm{NIH}$ R01 ES028104, and VA Merit Award 101 BX004161 awarded to Michelle L. Block. Veteran serum samples were provided through a CDMRP GWI consortium award (W81XWH-13-2-0072) to Dr. Kimberly Sullivan. The contents do not reflect the views of the US Department of Veteran's Affairs nor the United States Government.

\section{COMPETING INTERESTS}

The authors declare no competing interests.

\section{ADDITIONAL INFORMATION}

Supplementary information The online version contains supplementary material available at https://doi.org/10.1038/s41398-021-01517-1.

Correspondence and requests for materials should be addressed to M.L.B.

Reprints and permission information is available at http://www.nature.com/ reprints

Publisher's note Springer Nature remains neutral with regard to jurisdictional claims in published maps and institutional affiliations. Attribution 4.0 International License, which permits use, sharing, adaptation, distribution and reproduction in any medium or format, as long as you give appropriate credit to the original author(s) and the source, provide a link to the Creative Commons license, and indicate if changes were made. The images or other third party material in this article are included in the article's Creative Commons license, unless indicated otherwise in a credit line to the material. If material is not included in the article's Creative Commons license and your intended use is not permitted by statutory regulation or exceeds the permitted use, you will need to obtain permission directly from the copyright holder. To view a copy of this license, visit http://creativecommons. org/licenses/by/4.0/.

(c) The Author(s) 2021 